\title{
Noise-Improved Bayesian Estimation With Arrays of One-Bit Quantizers
}

\author{
David Rousseau and François Chapeau-Blondeau
}

\begin{abstract}
A noisy input signal is observed by means of a parallel array of one-bit threshold quantizers, in which all the quantizer outputs are added to produce the array output. This parsimonious signal representation is used to implement an optimal Bayesian estimation from the output of the array. Such conditions can be relevant for fast real-time processing in large-scale sensor networks. We demonstrate that, for input signals of arbitrary amplitude, the performance in the estimation can be improved by the addition of independent noises onto the thresholds in the array. These results constitute a novel instance of the phenomenon of suprathreshold stochastic resonance in arrays, by which nonlinear transmission or processing of signals with arbitrary amplitude can be improved through cooperative coupling with noise.
\end{abstract}

Index Terms-Estimation, noise, nonlinear arrays, quantizer, sensor arrays, stochastic resonance (SR).

\section{INTRODUCTION}

$\mathbf{M}$ ULTISENSOR arrays are currently an area of active research: Nonlinear arrays, microarrays or nanoarrays, intelligent sensing arrays incorporating preprocessing of data, and arrays drawing inspiration from sensory neurons constitute interesting lines of research in this area [1], [2]. In the present paper, we consider parallel arrays of threshold comparators or one-bit quantizers. Such simple devices can be useful in building large-scale arrays that are very efficient in terms of resources and time for data processing, storage, and communication, and in terms of energy supply, with a possibly high density of integration in solid-state realizations. Such arrays of comparators have similarities with flash analog-to-digital converters or with digital sonar arrays [3]. Such arrays of threshold devices also mimic, in a crude way, the nonlinear behavior, which can be present in networks of sensory neurons [4]. These arrays are therefore specially appealing in devising novel strategies based on multisensor networks for nonlinear signal and information processing.

A specific interest of such parallel arrays of comparators is that they have recently been shown to lend themselves to the phenomenon of suprathreshold stochastic resonance (SR). SR is a nonlinear phenomenon by which the action of noise can improve the performance of signal-processing or measurement systems [5]-[12]. Since its introduction some 20 years ago, SR has gradually been shown to be feasible under several different forms, with various types of systems, signals, and indexes of performance receiving improvement from the noise [10], [13]-[21]. Most occurrences of SR known today involve a

Manuscript received October 18, 2005; revised April 2, 2007.

The authors are with the Laboratoire d'Ingénierie des Systèmes Automatisés (LISA), Université d'Angers, 49000 Angers, France.

Digital Object Identifier 10.1109/TIM.2007.908125 signal which is, by itself, too small or ill-conditioned to elicit a strong response from a nonlinear system. Injection of noise then, through a cooperative interaction, brings assistance to the small signal in eliciting a more efficient response from the nonlinear system, for instance, by overcoming a threshold or a potential barrier.

Recently, an interesting new form of SR has been introduced under the name of suprathreshold SR since it is not restricted to a small, subthreshold, or ill-conditioned signal [22], [23]. Suprathreshold SR relies on a parallel array of identical nonlinear devices. At the location of each device, an independent noise is injected in the process. The result is to make each one of the identical devices elicit a distinct output in response to a same common input signal. When the individual outputs are collected or averaged over the array to produce a global response, it turns out that a net improvement can be obtained compared to the response of a single device with no noise injected. Qualitatively, its benefit comes from the diversity induced by the injected noises in the responses of the individual nonlinear devices over the array. This suprathreshold SR does not involve a small signal receiving assistance from the noise to elicit a more efficient response from a single isolated nonlinear system. As such, suprathreshold SR can be viewed as a specifically distinct mechanism under which an improvement by noise can take place.

Since its recent introduction in [22], suprathreshold SR has been shown to be possible in various conditions, with different types of signals and indexes of performance, including Shannon mutual information [3], [22], [23], input-output cross correlation [24], Fisher information [25], or signal-to-noise ratio [26]. Suprathreshold SR has also been applied to arrays of sensory neurons [27], to motion detectors [28], and to cochlear implants [29]. The most simple systems which have been reported to give way to suprathreshold SR and which have been exploited to investigate its properties are the parallel arrays of threshold comparators or quantizers mentioned earlier. A very recent study [30] demonstrates that such arrays can show detection capabilities that are improved by noise via suprathreshold SR. In the present paper, we extend the investigation of the capabilities of these arrays for noise-improved information processing or measurement via suprathreshold SR. We use these arrays for a Bayesian estimation task, and we demonstrate the possibility of conditions where the estimation performance is augmented by injection of noise in the array.

\section{Estimation From a Nonlinear Parallel ArRay}

A signal $x_{a}(t)$ is dependent upon parameter $a$, whose possible values are distributed according to the prior probability 
density function (pdf) $p_{a}(u)$. This signal $x_{a}(t)$ is observed by means of a parallel array of $N$ threshold comparators or one-bit quantizers, following the setting of [3] and [22]. We arrange for the possibility of a noise $\eta_{i}(t)$, which is independent of $x_{a}(t)$, to be added to $x_{a}(t)$ before quantization by quantizer $i$. The $N$ threshold noises $\eta_{i}(t)$ are taken as white, mutually independent, and identically distributed with cumulative distribution function $F_{\eta}(u)$. Quantizer $i$, with a threshold $\theta_{i}$, delivers the output

$$
y_{i}(t)=U\left[x_{a}(t)+\eta_{i}(t)-\theta_{i}\right], \quad i=1,2, \ldots, N
$$

where $U(u)$ is the Heaviside function, i.e., $U(u)=1$ if $u>0$ and is zero otherwise. The response $Y(t)$ of the array is obtained by summing the outputs of all the quantizers, as

$$
Y(t)=\sum_{i=1}^{N} y_{i}(t)
$$

The array output of (2) is measured at $M$ distinct times $t_{j}$, for $j=1$ to $M$, so as to provide $M$ data points $Y_{j}=Y\left(t_{j}\right)$. We then want to use the data $\boldsymbol{Y}=\left(Y_{1}, \ldots, Y_{M}\right)$ to estimate the value of $a$ that produced the observation. Once $\boldsymbol{Y}$ is observed, a posterior pdf $p(a \mid \boldsymbol{Y})$ for parameter $a$ can be defined. A given estimator $\widehat{a}(\boldsymbol{Y})$ for $a$ achieves a mean-square estimation error defined [31] as the expectation (conditioned by observation $\boldsymbol{Y}$ )

$$
\mathcal{E}(\boldsymbol{Y})=E\left[(a-\widehat{a})^{2} \mid \boldsymbol{Y}\right]=\int_{-\infty}^{\infty}[a-\widehat{a}(\boldsymbol{Y})]^{2} p(a \mid \boldsymbol{Y}) d a .
$$

It is easy to show that $\mathcal{E}(\boldsymbol{Y})$ of (3) can equivalently be expressed as

$$
\mathcal{E}(\boldsymbol{Y})=[\widehat{a}-\mathrm{E}(a \mid \boldsymbol{Y})]^{2}+\operatorname{var}(a \mid \boldsymbol{Y})
$$

with $E(a \mid \boldsymbol{Y})=\int_{-\infty}^{\infty} a p(a \mid \boldsymbol{Y}) d a$ and $\operatorname{var}(a \mid \boldsymbol{Y})=\int_{-\infty}^{\infty}[a-$ $E(a \mid \boldsymbol{Y})]^{2} p(a \mid \boldsymbol{Y}) d a$.

Since $\operatorname{var}(a \mid \boldsymbol{Y})$ in (4) is nonnegative and independent of $\widehat{a}$, the optimal Bayesian estimator that minimizes error $\mathcal{E}(\boldsymbol{Y})$, for any given observation $\boldsymbol{Y}$, comes out as

$$
\widehat{a}_{\mathrm{B}}(\boldsymbol{Y})=\mathrm{E}(a \mid \boldsymbol{Y})=\int_{-\infty}^{\infty} a p(a \mid \boldsymbol{Y}) d a
$$

and its performance is measured by the minimal error

$$
\mathcal{E}_{\mathrm{B}}(\boldsymbol{Y})=\operatorname{var}(a \mid \boldsymbol{Y})=\int_{-\infty}^{\infty}[a-E(a \mid \boldsymbol{Y})]^{2} p(a \mid \boldsymbol{Y}) d a .
$$

A model that shows how $x_{a}(t)$, and then $\boldsymbol{Y}$ via (1) and (2), are related to parameter $a$ allows one to define the conditional probabilities $\operatorname{Pr}\{\boldsymbol{Y} \mid a\}$ of observing one specific $\boldsymbol{Y}$ among the $(N+1)^{M}$ accessible states, given $a$. With the prior information summarized by $p_{a}(a)$, the Bayes rule then provides access to the posterior pdf under the form

$$
p(a \mid \boldsymbol{Y})=\frac{\operatorname{Pr}\{\boldsymbol{Y} \mid a\} p_{a}(a)}{\operatorname{Pr}\{\boldsymbol{Y}\}}
$$

with the total probability $\operatorname{Pr}\{\boldsymbol{Y}\}=\int_{-\infty}^{\infty} \operatorname{Pr}\{\boldsymbol{Y} \mid a\} p_{a}(a) d a$.
For any given observation $Y$, the optimal Bayesian estimator $\widehat{a}_{\mathrm{B}}(\boldsymbol{Y})$ from (5) achieves the minimum $\mathcal{E}_{\mathrm{B}}(\boldsymbol{Y})$ from (6) of the error $\mathcal{E}(\boldsymbol{Y})$ from (3). Consequently, $\widehat{a}_{\mathrm{B}}(\boldsymbol{Y})$ also achieves the minimum $\overline{\mathcal{E}}_{\mathrm{B}}$ of error $\mathcal{E}(\boldsymbol{Y})$ averaged over every possible observation $\boldsymbol{Y}$, i.e., $\widehat{a}_{\mathrm{B}}(\boldsymbol{Y})$ minimizes $\sum_{\boldsymbol{Y}} \mathcal{E}(\boldsymbol{Y}) \operatorname{Pr}\{\boldsymbol{Y}\}$, and the minimum that is reached is

$$
\overline{\mathcal{E}}_{\mathrm{B}}=\sum_{\boldsymbol{Y}} \operatorname{var}(a \mid \boldsymbol{Y}) \operatorname{Pr}\{\boldsymbol{Y}\}
$$

where the sums on $\boldsymbol{Y}$ run over the $(N+1)^{M}$ accessible states, from $\boldsymbol{Y}=\left(Y_{1}=0, \ldots, Y_{M}=0\right)$ to $\boldsymbol{Y}=\left(Y_{1}=\right.$ $\left.N, \ldots, Y_{M}=N\right)$.

To proceed, at any time $t_{j}$, for a fixed given value $x_{a}$ of the input signal $x_{a}\left(t_{j}\right)$, we have the conditional probability $\operatorname{Pr}\left\{y_{i}\left(t_{j}\right)=0 \mid x_{a}\right\}$ which is also $\operatorname{Pr}\left\{x_{a}+\eta_{i}\left(t_{j}\right) \leq \theta_{i}\right\}$. This amounts to

$$
\operatorname{Pr}\left\{y_{i}\left(t_{j}\right)=0 \mid x_{a}\right\}=F_{\eta}\left(\theta_{i}-x_{a}\right) .
$$

In the same way, we have $\operatorname{Pr}\left\{y_{i}\left(t_{j}\right)=1 \mid x_{a}\right\}=1-F_{\eta}\left(\theta_{i}-x_{a}\right)$.

To show the possibility of a noise-improved estimation, we assume, as done in [23], the simplest configuration of the array, where all the thresholds $\theta_{i}$ share the same value $\theta_{i}=\theta$ for all $i$. The conditional probability $\operatorname{Pr}\left\{Y\left(t_{j}\right)=Y_{j} \mid x_{a}\right\}$, for any accessible value $Y_{j}=0$ to $N$, then follows, according to the binomial distribution, as

$$
\begin{aligned}
\operatorname{Pr}\left\{Y\left(t_{j}\right)\right. & \left.=Y_{j} \mid x_{a}\right\} \\
& =C_{Y_{j}}^{N}\left[1-F_{\eta}\left(\theta-x_{a}\right)\right]^{Y_{j}} F_{\eta}\left(\theta-x_{a}\right)^{N-Y_{j}}
\end{aligned}
$$

where $C_{Y_{j}}^{N}$ is the binomial coefficient.

For the sake of definiteness, concerning the parametric dependence of $x_{a}(t)$ on $a$, we shall consider the broad class of processes, where $x_{a}(t)$ is formed by the additive mixture $x_{a}(t)=\xi(t)+s_{a}(t)$. The signal $\xi(t)$ is a random input noise, which is white and independent of $a$ and of $\eta_{i}$ s, with pdf $f_{\xi}(u)$. Signal $s_{a}(t)$ is deterministic for a fixed value of $a$. For instance, $s_{a}(t)$ may be the constant signal $s_{a}(t) \equiv a$ or the periodic signal $s_{a}(t)=\sin (a t)$, or any other signal of known deterministic form that is parameterized by $a$. At time $t$, the pdf for the values of $x_{a}(t)$ given $a$ is thus given as $f_{\xi}\left[u-s_{a}(t)\right]$, and we therefore obtain the conditional probability

$$
\begin{aligned}
\operatorname{Pr}\left\{Y\left(t_{j}\right)=Y_{j} \mid a\right\} & =\int_{-\infty}^{\infty} C_{Y_{j}}^{N}\left[1-F_{\eta}(\theta-u)\right]^{Y_{j}} \\
& \times F_{\eta}(\theta-u)^{N-Y_{j}} f_{\xi}\left(u-s_{a}\left(t_{j}\right)\right) d u .
\end{aligned}
$$

Since the noises $\xi(t)$ and $\eta_{i}(t)$ are white and mutually independent, the conditional probability of (7) factorizes as

$$
\operatorname{Pr}\{\boldsymbol{Y} \mid a\}=\prod_{j=1}^{M} \operatorname{Pr}\left\{Y\left(t_{j}\right)=Y_{j} \mid a\right\} .
$$

Equations (11) and (12), through (7), will enable an explicit evaluation of the optimal Bayesian estimator of (5) and of its performance measured by (6) or (8). This will be obtainable, 


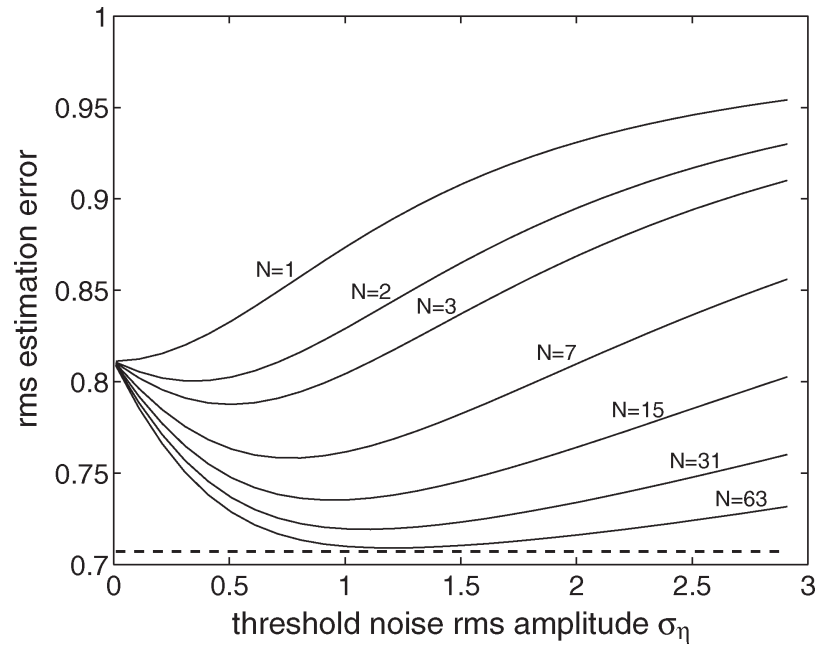

Fig. 1. RMS error of the optimal Bayesian estimator as a function of the rms amplitude $\sigma_{\eta}$ of the threshold noises $\eta_{i}(t)$ chosen zero-mean Gaussian. The input noise $\xi(t)$ is a zero-mean Gaussian with an rms amplitude $\sigma_{\xi}=1$. The prior pdf $p_{a}(u)$ is a zero-mean Gaussian with an rms amplitude $\sigma_{a}=1$. All the thresholds in the array are set to $\theta=0$, with $M=1$. The solid lines are $\overline{\mathcal{E}}_{\mathrm{B}}^{1 / 2}$ from the theory of (8). The dashed line is the estimation error $\sigma_{\mathrm{Bin}}$ from (13) of the same optimal (minimal error) estimator operating directly on the input-signal-noise mixture $x_{a}(t)$.

possibly through numerical integration, for any definite conditions concerning the following: 1$)$ the input signal via $s_{a}(t)$ and $\left.p_{a}(u) ; 2\right)$ the input white noise via $f_{\xi}(u)$, and 3$)$ the measuring array via $\theta, N$, and $F_{\eta}(u)$. We shall now use this theory to illustrate the existence of conditions where enhancement of the threshold noises $\eta_{i}(t)$ results in an improved performance for the optimal Bayesian estimator of $a$ from $\boldsymbol{Y}$.

\section{NOISE-IMPROVED ESTIMATION}

For illustration of the possibility of a noise-improved estimation via suprathreshold SR, we consider the simple case of a constant input signal $s_{a}(t) \equiv a$. The input noise $\xi(t)$ is taken with zero mean and rms amplitude $\sigma_{\xi}$. The common threshold in the array is set at the input mean, i.e., $\theta=E\left[x_{a}(t)\right]$, which is known as soon as $p_{a}(u)$ and $f_{\xi}(u)$ are specified. Fig. 1 then shows the evolution of the rms estimation error $\overline{\mathcal{E}}_{\mathrm{B}}^{1 / 2}$ from (8) as a function of the rms amplitude $\sigma_{\eta}$ of the threshold noises for various sizes $N$ of the array when both the input noise $\xi(t)$ and the threshold noises $\eta_{i}(t)$ are zero-mean Gaussian.

In Fig. 1, the choice $\theta=E\left[x_{a}(t)\right]$ ensures that the inputsignal-noise mixture $x_{a}(t)$ is suprathreshold, in the sense that $x_{a}(t)$ evolves on both sides of the threshold $\theta$ and can cross $\theta$ without assistance from the threshold noises $\eta_{i}(t)$. As a consequence, if the array is reduced to a single device $(N=1)$, the added threshold noise $\eta_{1}(t)$ does not improve the performance, and its increase monotonically degrades the estimation error $\overline{\mathcal{E}}_{\mathrm{B}}^{1 / 2}$, as shown in Fig. 1 . When $N>1$, in the absence of the threshold noises $\eta_{i}(t)$, all the quantizers in the array switch in unison, acting like a single quantizer. In this case, at $\sigma_{\eta}=0$, the performance of the array does not depend on size $N$ of the array. At $N>1$, application of the threshold noises $\eta_{i}(t)$ then allows for the quantizers to differently respond. This is the source of the richer response of the array with threshold noises. For the estimation task performed from the array output, this translates into a possibility of improving the performance measured by the estimation error $\overline{\mathcal{E}}_{\mathrm{B}}^{1 / 2}$, with, for each $N>1$, a nonzero optimal amount of the threshold noises that minimizes $\overline{\mathcal{E}}_{\mathrm{B}}^{1 / 2}$, as shown in Fig. 1. This is the suprathreshold SR effect, which is reported here in a Bayesian estimation task.

For comparison of the optimal Bayesian estimation from the array output that is addressed in Fig. 1, it is interesting to consider the same optimal estimator (the minimal-error estimator) that would directly operate on the input-signal-noise mixture $x_{a}(t)$ rather than on its quantized representation by the array. By applying on $x_{a}(t)$ the principles of optimal Bayesian estimation similar to those exposed in Section II, it comes out that, when the input noise $\xi(t)$ is zero-mean Gaussian, the optimal Bayesian estimator [31] operating directly on $x_{a}(t)$ is $\widehat{a}_{\text {Bin }}$, which achieves the rms estimation error $\sigma_{\text {Bin }}$, verifying

$$
\frac{1}{\sigma_{\mathrm{Bin}}^{2}}=\frac{1}{\sigma_{a}^{2}}+\frac{M}{\sigma_{\xi}^{2}}
$$

with the estimator itself reading

$$
\widehat{a}_{\mathrm{Bin}}=\frac{\sigma_{\mathrm{Bin}}^{2}}{\sigma_{a}^{2}} \mathrm{E}(a)+M \frac{\sigma_{\mathrm{Bin}}^{2}}{\sigma_{\xi}^{2}} \bar{x}_{a}
$$

with the empirical mean of the measurements $\bar{x}_{a}=$ $M^{-1} \sum_{j=1}^{M} x_{a}\left(t_{j}\right)$.

In Fig. 1, the rms estimation error $\sigma_{\mathrm{Bin}}$ from (13) is represented by the dashed line. It is shown in Fig. 1 that, in the optimal Bayesian estimation from the array output, the minimal value reached by the estimation error $\overline{\mathcal{E}}_{\mathrm{B}}^{1 / 2}$ at the optimal level of the threshold noises, as $N$ increases, tends to the performance $\sigma_{\mathrm{Bin}}$ of the optimal Bayesian estimator operating directly on the input-signal-noise mixture $x_{a}(t)$. This proves that the optimal Bayesian estimator $\widehat{a}_{\mathrm{B}}$ from the output of the array of one-bit quantizers, as the array becomes large, is able to perform as efficiently as the optimal Bayesian estimator $\widehat{a}_{\text {Bin }}$ operating directly on the input-signal-noise mixture $x_{a}(t)$. At the same time, Fig. 1 shows that, with relatively modest sizes $N$, the performance of the array comes close to the best performance $\sigma_{\mathrm{Bin}}$ at the input. Advantages afforded by the array lie in the parsimony of the representation and simplicity of operation (possibly associated to rapidity), working on a few bits collected by the comparators, as opposed to the infinite number of bits, in principle, associated with the analog input $x_{a}(t)$. In addition, in various circumstances, it may be the case that the analog input $x_{a}(t)$ is not directly accessible when no linear transducers are available to form a direct image of $x_{a}(t)$ that is manageable by the signal-processing system.

Fig. 2 represents the evolution of the rms estimation error $\overline{\mathcal{E}}_{\mathrm{B}}^{1 / 2}$ in the same conditions as in Fig. 1, except for the number of data points $M=2$. As usual, in Bayesian estimation, the theoretical calculation of the rms estimation error gets more and more computationally demanding as the number of data points $M$ increases. This is because the rms error results from an average (expressed by multiple sums or integrals) over all 


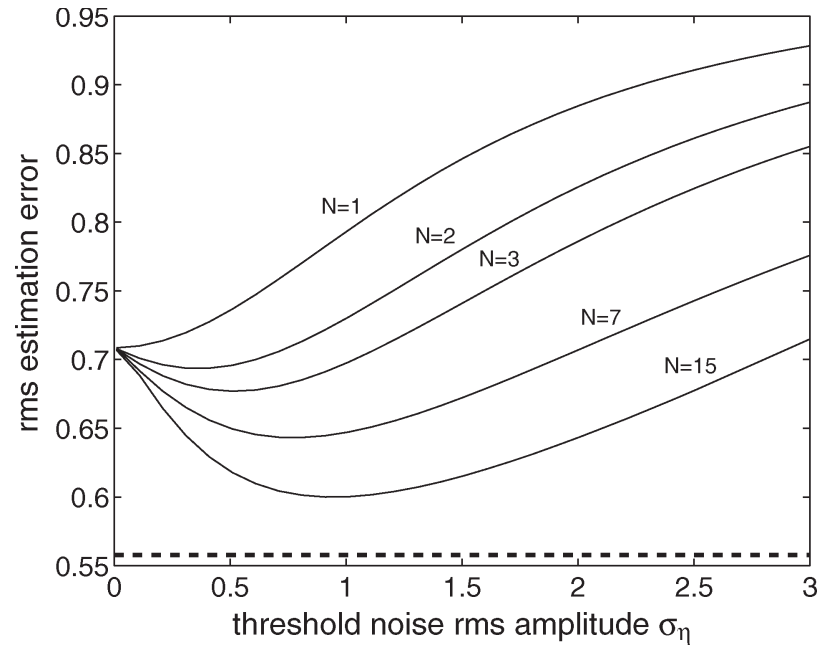

Fig. 2. Same as in Fig. 1, except that $M=2$.

possible configurations of the data, with the number of that configurations exponentially growing with the number of data points $M$. This is the computational price to pay to have access to the theoretical performance of a Bayesian estimator. However, the practical application of the estimator itself on one observed data set is much more direct since this does not require averaging over all the data configurations. This explains why our calculation of the rms estimation error in Figs. 1 and 2 remains limited to small values of $M$. Nevertheless, the important point with Fig. 2 is to verify that the possibility of improvement by noise of the rms error $\overline{\mathcal{E}}_{\mathrm{B}}^{1 / 2}$ is preserved when the number $M$ of data points increases. The minimal rms error (shown in Fig. 2 in arrays with size $N>1$ ) is always achieved at a nonzero level of the added threshold noises $\eta_{i}(t)$, which is the SR effect.

\section{Discussion AND CONCLUSION}

To summarize, we have considered simple threshold comparators for estimation in a Bayesian framework. When such simple binary sensors are used in isolation, a rather crude estimation capability results. We have shown that, by associating these simple sensors into arrays, an enhanced estimation capability is obtained, owing to the action of added noises, through the SR phenomenon. The present results contribute to the inventory and analysis of SR, especially through the specific mechanism of SR in parallel uncoupled arrays. They also contain significance in the direction of novel architectures and processes for sensing arrays.

The results that are shown in Figs. 1 and 2 represent a new manifestation of suprathreshold SR, which is applied here for the first time to a Bayesian estimation task. The evolutions of the estimation error obtained in Figs. 1 and 2 are quite reminiscent of the evolutions of other performance indexes obtained in different instances of suprathreshold SR, namely, the Shannon mutual information in [23], the correlation coefficient in [24], the Fisher information in [25], a signal-to-noise ratio in [26], or a probability of detection error in [30]. This is an important point to note because all these indexes of performance are different and relate to distinct signal-processing tasks. This contributes to establish SR, which is understood as a possibility of noise-improved signal processing, as a general nonlinear phenomenon which can be obtained in many contexts under many distinct forms, with the ability to enhance many different performance indexes. The specific structure considered here for Bayesian estimation, i.e., parallel arrays of one-bit quantizers, can be considered as realizing a suboptimal estimator. The central contribution of the present study is to demonstrate the feasibility in this structure, for improvement by noise of Bayesian estimation. A common feature with many other forms of SR previously reported is the suboptimal character of the processor which receives improvement by noise, as stressed, for instance, in [32]. However, some optimal processors have also been shown to lend themselves to a form of SR, with a performance for the optimal processor which can improve when the optimal processor operates at a higher level of noise. This possibility of SR has been shown with optimal detectors [33]-[35] and optimal estimators [12], and the mechanism of SR in parallel arrays, as studied here, could be investigated for further improvement in this direction.

The present approach is based on SR for improvement by addition of the noises $\eta_{i}(t)$ to one-bit quantizers with a fixed common threshold. The procedure can also be viewed as quantization with a distribution of random thresholds. By contrast, the common approach to quantization would be to select a nonrandom distribution of the thresholds in order to optimize a specific design criterion, and many studies have been conducted along this line for optimal quantization [36]. However, finding optimal distributions of the thresholds is, generally, a difficult optimization problem; moreover, the solutions are likely to vary with the properties of the input signal $s_{a}(t)$ and input noise $\xi(t)$. Addition of the noises $\eta_{i}(t)$ with a common threshold can be considered as an SR-based alternative that dispenses one from facing the difficult and specific determination of the optimal distribution of the quantization thresholds. A good performance can, nevertheless, be obtained by this SR-based approach with a common threshold and added noises $\eta_{i}(t)$, as proved by the behavior of the performance of the array compared to the overall best performance given by $\sigma_{\operatorname{Bin}}$ in Figs. 1 and 2. The SR-based estimation might also reveal more robust to variations in the input $x_{a}(t)$ with respect to the nominal conditions of an optimal distribution of the quantization thresholds. Finally, situations may exist due to hardware limitations, where the thresholds $\theta_{i}$ are not separately adjustable; this could be the case, for instance, with nanodevices or with sensory neurons. However, the more complex conditions with distributed thresholds $\theta_{i}$ could be explicitly investigated through an extension of the present approach to uncover how the specific properties and potentialities of the noise-improved estimation evolve. Multiple parameter estimation could also be addressed as an extension to the present approach.

Compared to linear sensors, the present approach is based on quite simple nonlinear devices producing a very parsimonious one-bit representation of the signal. Estimation aided by noise with such one-bit quantizers can be specially appealing in the context of measurement for real-time processing with largescale sensor networks. In addition, in various circumstances, the 
analog input may not be accessible when no linear transducers are available for this. It will be the case, for instance, with sensory neurons, which form a thresholded all-or-nothing type of representation of an analog signal from the physical environment, bearing similarities with the representation by the present array of comparators [27], [29], [37]. This will also be the case with nanodevices with intrinsic nonlinearities and usable in building sensor microarrays [38]. The estimation aided by noise that we have described in arrays can be specially relevant in these contexts of intrinsically nonlinear sensing networks. This can provide a basis for devising novel unconventional intelligent sensing arrays that are capable of exploiting the noise.

\section{REFERENCES}

[1] O. Kanoun and H. R. Tränkler, "Sensor technology advances and future trends," IEEE Trans. Instrum. Meas., vol. 53, no. 6, pp. 1497-1501, Dec. 2004.

[2] J. S. Wilson, Sensor Technology Handbook. Boston, MA: Newnes, 2004.

[3] M. D. McDonnell, D. Abbott, and C. E. M. Pearce, "An analysis of noise enhanced information transmission in an array of comparators," Microelectron. J., vol. 33, no. 12, pp. 1079-1089, Dec. 2002.

[4] M. A. Arbib, The Handbook of Brain Theory and Neural Networks. Cambridge, MA: MIT Press, 2002.

[5] L. Gammaitoni, P. Hänggi, P. Jung, and F. Marchesoni, "Stochastic resonance," Rev. Modern Phys., vol. 70, no. 1, pp. 223-287, Jan. 1998.

[6] B. Andò, S. Baglio, S. Graziani, and N. Pitrone, "Optimal improvement in bistable measurement device performance via stochastic resonance," Int. J. Electron., vol. 86, no. 7, pp. 791-806, Jul. 1999.

[7] B. Andò and S. Graziani, Stochastic Resonance: Theory and Applications. Boston, MA: Kluwer, 2000.

[8] B. Andò, S. Baglio, S. Graziani, and N. Pitrone, "Measurements of parameters influencing the optimal noise level in stochastic systems," IEEE Trans. Instrum. Meas., vol. 49, no. 5, pp. 1137-1143, Oct. 2000.

[9] B. Andò and S. Graziani, "Adding noise to improve measurement," IEEE Instrum. Meas. Mag., vol. 4, no. 1, pp. 24-30, Mar. 2001.

[10] G. P. Harmer, B. R. Davis, and D. Abbott, "A review of stochastic resonance: Circuits and measurement," IEEE Trans. Instrum. Meas., vol. 51, no. 2, pp. 299-309, Apr. 2002.

[11] A. Nikitin, N. G. Stocks, and A. R. Bulsara, "Signal detection via residence times statistics: Noise-mediated minimization of the measurement error," Phys. Rev. E, Stat. Phys. Plasmas Fluids Relat. Interdiscip. Top., vol. 68, no. 3, pp. $036133-1-036133-4$, Sep. 2003.

[12] F. Chapeau-Blondeau and D. Rousseau, "Noise-enhanced performance for an optimal Bayesian estimator," IEEE Trans. Signal Process., vol. 52, no. 5, pp. 1327-1334, May 2004.

[13] S. Mitaim and B. Kosko, "Adaptive stochastic resonance," Proc. IEEE, vol. 86, no. 11, pp. 2152-2183, Nov. 1998.

[14] G. P. Harmer and D. Abbott, "Simulation of circuits demonstrating stochastic resonance," Microelectron. J., vol. 31, no. 7, pp. 553-559, Jul. 2000.

[15] J. J. Collins, C. C. Chow, and T. T. Imhoff, "Stochastic resonance without tuning," Nature, vol. 376, no. 6537, pp. 236-238, Jul. 1995.

[16] Z. Gingl, L. B. Kiss, and F. Moss, "Non-dynamical stochastic resonance: Theory and experiments with white and arbitrarily coloured noise," Europhys. Lett., vol. 29, no. 3, pp. 191-196, Jan. 1995.

[17] M. E. Inchiosa and A. R. Bulsara, "Signal detection statistics of stochastic resonators," Phys. Rev. E, Stat. Phys. Plasmas Fluids Relat. Interdiscip. Top., vol. 53, no. 3, pp. R2 021-R2 024, Mar. 1996.

[18] X. Godivier, J. Rojas-Varela, and F. Chapeau-Blondeau, "Noise-assisted signal transmission via stochastic resonance in a diode nonlinearity," Electron. Lett., vol. 33, no. 20, pp. 1666-1668, Sep. 1997.

[19] D. G. Luchinsky, R. Mannella, P. V. E. McClintock, and N. G. Stocks, "Stochastic resonance in electrical circuits-I: Conventional stochastic resonance," IEEE Trans. Circuits Syst. II, vol. 46, no. 9, pp. 1205-1214, Sep. 1999.

[20] F. Chapeau-Blondeau, "Noise-assisted propagation over a nonlinear line of threshold elements," Electron. Lett., vol. 35, no. 13, pp. 1055-1056, Jun. 1999.

[21] S. Zozor and P. O. Amblard, "On the use of stochastic resonance in sine detection," Signal Process., vol. 82, no. 3, pp. 353-367, Mar. 2002.

[22] N. G. Stocks, "Suprathreshold stochastic resonance in multilevel threshold systems," Phys. Rev. Lett., vol. 84, no. 11, pp. 2310-2313, Mar. 2000.
[23] N. G. Stocks, "Information transmission in parallel threshold arrays: Suprathreshold stochastic resonance," Phys. Rev. E, Stat. Phys. Plasmas Fluids Relat. Interdiscip. Top., vol. 63, no. 4, pp. 041114-1-041114-9, Apr. 2001.

[24] M. D. McDonnell, D. Abbott, and C. E. M. Pearce, "A characterization of suprathreshold stochastic resonance in an array of comparators by correlation coefficient," Fluctuation Noise Lett., vol. 2, no. 3, pp. L205-L220, 2002.

[25] D. Rousseau, F. Duan, and F. Chapeau-Blondeau, "Suprathreshold stochastic resonance and noise-enhanced Fisher information in arrays of threshold devices," Phys. Rev. E, Stat. Phys. Plasmas Fluids Relat. Interdiscip. Top., vol. 68, no. 3, pp. 031107-1-031107-10, Sep. 2003.

[26] D. Rousseau and F. Chapeau-Blondeau, "Suprathreshold stochastic resonance and signal-to-noise ratio improvement in arrays of comparators," Phys. Lett. A, vol. 321, no. 5/6, pp. 280-290, Feb. 2004.

[27] N. G. Stocks and R. Mannella, "Generic noise-enhanced coding in neuronal arrays," Phys. Rev. E, Stat. Phys. Plasmas Fluids Relat. Interdiscip. Top., vol. 64, no. 3, pp. 030902-1-030902-4, Sep. 2001.

[28] G. P. Harmer and D. Abbott, "Motion detection and stochastic resonance in noisy environments," Microelectron. J., vol. 32, no. 12, pp. 959-967, Dec. 2001.

[29] N. G. Stocks, D. Allingham, and R. P. Morse, "The application of suprathreshold stochastic resonance to cochlear implant coding," Fluctuation Noise Lett., vol. 2, no. 3, pp. L169-L181, 2002.

[30] D. Rousseau and F. Chapeau-Blondeau, "Constructive role of noise in signal detection from parallel arrays of quantizers," Signal Process., vol. 85, no. 3, pp. 571-580, Mar. 2005.

[31] S. M. Kay, Fundamentals of Statistical Signal Processing: Estimation Theory. Englewood Cliffs, NJ: Prentice-Hall, 1993.

[32] S. Kay, "Can detectability be improved by adding noise?" IEEE Signal Process. Lett., vol. 7, no. 1, pp. 8-10, Jan. 2000.

[33] F. Chapeau-Blondeau, "Stochastic resonance for an optimal detector with phase noise," Signal Process., vol. 83, no. 3, pp. 665-670, Mar. 2003.

[34] D. Rousseau and F. Chapeau-Blondeau, "Stochastic resonance and improvement by noise in optimal detection strategies," Digit. Signal Process., vol. 15, no. 1, pp. 19-32, Jan. 2005.

[35] F. Chapeau-Blondeau and D. Rousseau, "Constructive action of additive noise in optimal detection," Int. J. Bifurc. Chaos, vol. 15, no. 9, pp. $2985-$ 2994, 2005

[36] R. M. Gray and D. L. Neuhoff, "Quantization," IEEE Trans. Inf. Theory, vol. 44, no. 6, pp. 2325-2383, 1998.

[37] M. D. McDonnell and D. Abbott, "Open questions for suprathreshold stochastic resonance in sensory neural models for motion detection using artificial insect vision," in Proc. AIP Conf., 2003, vol. 665, pp. L51-L58.

[38] I. Y. Lee, X. Liu, B. Kosko, and C. Zhou, "Nanosignal processing: Stochastic resonance in carbon nanotubes that detect subthreshold signals," Nano Lett., vol. 3, no. 12, pp. 1683-1686, 2003.

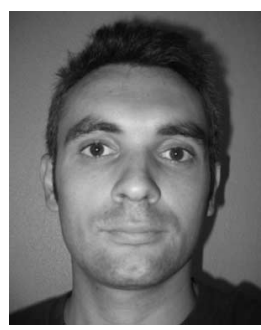

David Rousseau was born in Le Mans, France, in 1973. He received the M.S. degree in acoustics and signal processing from the Institut de Recherche Coordination Acoustique et Musique, Paris, France, in 1996 and the Ph.D. degree in nonlinear signal processing from the Université d'Angers, Angers, France, in 2004

He is currently a Maitre de Conférences with the Université d'Angers.

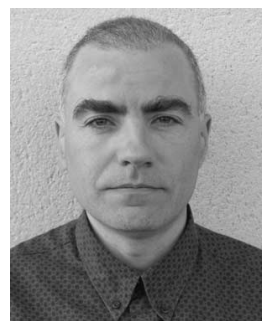

François Chapeau-Blondeau was born in France in 1959. He received the Engineer Diploma from ESEO, Angers, France, in 1982, the Ph.D. degree in electrical engineering from University Paris 6, Paris, France, in 1987, and the Habilitation degree from the Université d'Angers in 1994.

In 1988, he was a Research Associate with the Department of Biophysics, Mayo Clinic, Rochester, MN, working on biomedical ultrasonics. Since 1990, he has been with the Université d'Angers, where he is currently a Professor of electronic and information sciences. His research interests include nonlinear systems and signal processing, and the interface between physics and information sciences. 